\title{
Improvement of Fungal Cellulase Production by Mutation and Optimization of Solid State Fermentation
}

\author{
Van Hanh Vu' ${ }^{1,2}$, Tuan Anh Pham ${ }^{1}$ and Keun Kim ${ }^{1 *}$ \\ ${ }^{1}$ Department of Bioscience and Biotechnology, The University of Suwon, Hwaseong 445-743, Korea \\ ${ }^{2}$ Institute of Biotechnology, Vietnam Academy of Science and Technology, Hanoi 10600, Vietnam
}

(Received November 10, 2010. Accepted December 13, 2010)

Spores of Aspergillus sp. SU14 were treated repeatedly and sequentially with $\mathrm{Co}^{60} \gamma$-rays, ultraviolet irradiation, and Nmethyl-N'-nitro-N-nitrosoguanidine. One selected mutant strain, Aspergillus sp. SU14-M15, produced cellulase in a yield 2.2fold exceeding that of the wild type. Optimal conditions for the production of cellulase by the mutant fungal strain using solid-state fermentation were examined. The medium consisted of wheat-bran supplemented with $1 \%(\mathrm{w} / \mathrm{w})$ urea or $\mathrm{NH}_{4} \mathrm{Cl}$, $1 \%(\mathrm{w} / \mathrm{w})$ rice starch, $2.5 \mathrm{mM} \mathrm{MgCl}_{2}$, and $0.05 \%$ (v/w) Tween 80. Optimal moisture content and initial $\mathrm{pH}$ was $50 \%(\mathrm{v} / \mathrm{w})$ and 3.5, respectively, and optimal aeration area was 3/100 (inoculated wheat bran/container). The medium was inoculated with $25 \% 48 \mathrm{hr}$ seeding culture and fermented at $35^{\circ} \mathrm{C}$ for 3 days. The resulting cellulase yield was 8.5-fold more than that of the wild type strain grown on the basal wheat bran medium.

KEYWORDS : Aspergillus sp., Cellulase, Mutation, Optimization of solid state fermentation

The need for utilizing renewable resources to meet the future demand for fuel has increased the attention on cellulose, the most abundant and renewable resource in the world. Cellulose is degraded by cellulases to reducing sugars and is fermented by yeast or bacteria to ethanol [1], which is an attractive alternative fuel to petroleum. Ethanol production from glucose is most commonly carried out by the yeast Saccharomyces cerevisiae [2] and the bacterium Zymomonas mobilis [3], with another yeast species, Brettanomyces custersii, being useful in ethanol production from cellobiose [4]. Cellulases are produced by various fungi and bacteria. Trichoderma reesei is a popular source of commercial cellulose, as it displays high cellulose activity, owing to the high protein secretion capacity of mutant strains obtained by random mutagenesis [5]. Aspergillus sp. also has been widely exploited for production of cellulases [6, 7].

During ethanol production from lignocellulosics, cellulases play a very important role in the cellulose digestion process. However, the very prohibitive cost of cellulases due to the large amounts required for cellulose digestion hinders their widespread use $[1,8]$. Reduced cost of cellulase production, improved cellulase activity and an increase in sugar yields are all vital to reducing the processing costs of bioethanol from cellulosic substrates [9].

Mutagenic agents can achieve strain improvement [10]. Treatment of Fusarium oxysporum with ultraviolet (UV)

$\overline{\text { *Corresponding author }}<$ E-mail : kkim@suwon.ac.kr> radiation followed by $\mathrm{N}$-methyl-N'-nitro-N-nitrosoguanidine (NTG) has been used to improve cellulase production [11]. The simultaneous treatment of a fungus with NTG, UV and NTG combined with $\mathrm{Co}^{60} \gamma$-rays created a mutant that more exuberantly produced cellulose [12]. Both solid and liquid fermentation systems have been used for the production of cellulases. Solid state fermentation (SSF) is a process in which an insoluble substrate is fermented with sufficient moisture but without free water. The advantages of SSF compared to submerged liquid fermentation are numerous and include ease of use, lower capital cost, superior productivity, reduced energy requirement, use of simpler fermentation media, absence of rigorous control of fermentation parameters, reduced water requirement, reduced produced of wastewater, easier control of bacterial contamination and lower cost for downstream processing $[13,14]$.

To date, research concerning strain improvement and optimization of culture conditions has been done separately. To maximize cellulase production, strain improvement and optimization of culture conditions should be performed consequently. With this knowledge, the present study sought to modify a fungal strain and optimize solid medium and culture conditions to enhance cellulose production.

\section{Materials and Methods}

Fungal strain and media. Laboratory stock culture of Aspergillus sp. SU14, a hyper-producing cellulose origi- 
nally isolated from soil was used. The frozen culture stored at $-80^{\circ} \mathrm{C}$ was streaked on potato dextrose agar (PDA, 2\% agar) and cultured at $30^{\circ} \mathrm{C}$ for $4 \sim 7$ days.

Fungal strain improvement by mutagenesis. The spores of fungal strain SU14 $\left(10^{8}\right.$ spores $\left./ \mathrm{mL}\right)$ were harvested from 6-day-old spores grown on PDA following exposure to different doses $(0.5 \sim 2.5 \mathrm{KGy})$ of $\mathrm{Co}^{60} \gamma$-rays. The survivors were grown by spreading $0.1 \mathrm{~mL}$ of treated spores on PDA containing $0.1 \%(\mathrm{w} / \mathrm{v})$ cellulose azure and incubating at $30^{\circ} \mathrm{C}$ for $4 \sim 7$ days. Mutants evident by the presence of large clear zones around the colonies were selected and re-irradiated with $\mathrm{Co}^{60} \gamma$-rays. The best-surviving mutant was then exposed to $\mathrm{UV}$ radiation $(30 \mathrm{~W}$ at a distance of $50 \mathrm{~cm}$ for 2 60 min; present modifications) [11]. The selected UV mutant was treated with NTG $(100 \mu \mathrm{g} / \mathrm{mL}$ in $0.2 \mathrm{M}$ citrate buffer solution, $\mathrm{pH} 5)$ using four cycles of 10 60 min each. The mutant spores were washed four times with saline solution and spread on PDA as described above. After treatment with various mutagenic agents, 500 mutant strains were picked up and screened. The mutant strain that was superior in cellular production, which was designated SU14-M15, was used for further study.

Preparation of seeding culture. Ten grams of wheat bran powder and $90 \mathrm{~mL}$ distilled water in a $250 \mathrm{~mL}$ Erlenmeyer flask (final $\mathrm{pH} 3.5$ ), was sterilized at $121^{\circ} \mathrm{C}$ for $20 \mathrm{~min}$, and then cooled. To this, one plug $\left(1 \times 1 \mathrm{~cm}^{2}\right)$ of 7-day-old conidia-mycelia were inoculated and incubated at $30^{\circ} \mathrm{C}$ in an orbital shaking incubator operating at 200 rpm for 1 day.

Solid state fermentation (SSF). A $500 \mathrm{~mL}$ Erlenmeyer flask containing $20 \mathrm{~g}$ of wheat bran with $33.3 \%$ moisture was plugged with cotton and sterilized for $20 \mathrm{~min}$ at $121^{\circ} \mathrm{C}$. After cooling, $10 \%$ of a 1-day-old liquid culture was inoculated and incubated at $30^{\circ} \mathrm{C}$ for 3 days.

Preparation of crude cellulase. The moldy wheat-bran that developed after 3 days of fermentation was mixed with distilled water at a $1: 50(\mathrm{w} / \mathrm{v})$ ratio. The mixture was shaken at $30^{\circ} \mathrm{C}$ for $1 \mathrm{hr}$ in an orbital shaker operating at $200 \mathrm{rpm}$. The supernatant obtained after centrifugation of the mixture at 7,000 rpm for $10 \mathrm{~min}$ was used as crude enzyme source.

Cellulase activity assay. The activity of cellulase was determined as previously detailed [15]. The $1 \mathrm{~mL}$ enzyme reaction mixture was composed of $0.5 \mathrm{~mL}$ of diluted enzyme and $0.5 \mathrm{~mL}$ of $1 \%(\mathrm{w} / \mathrm{v})$ carboxymethyl cellulose (CMC; Sigma, St. Louis, MO, USA) in acetate buffer $(50 \mathrm{mM}, \mathrm{pH} 5)$. The reaction mixture was incubated at $50^{\circ} \mathrm{C}$ for $30 \mathrm{~min}$ and the released reducing sugar was determined by the 3,5-dinitrosalicylic acid method [16]. One unit (U) of enzyme activity was defined as the amount of enzyme required to liberate $1 \mu \mathrm{M}$ of glucose from $\mathrm{CMC}$ per min under the assay conditions. Cellulase activity was expressed as unit per $1 \mathrm{~g}$ of fermented solid wheat bran $(\mathrm{U} / \mathrm{g})$.

Optimization of SSF. SSF was carried out to study the effect of various physico-chemical parameters required for the maximal production of cellulase by SU14-M15. The parameters that were optimized were substrate (wheat bran, husk, saw dust), moisture (20 80\% v/w, where $\mathrm{v}$ and $\mathrm{w}$ represent water and water + dried wheat bran, respectively), incubation temperature $\left(20 \sim 45^{\circ} \mathrm{C}\right), \mathrm{pH}$ of solid culture (3 7), incubation time (2 6 days), aeration area (15 45 g of moistened wheat bran in a $500 \mathrm{~mL}$ Erlenmeyer flask, age of liquid culture (1 4 days), inoculum size $(10 \sim 50 \%, \mathrm{v} / \mathrm{w})$. Studies were also conducted to examine the effect on cellulose production of various additives supplemented into wheat bran solid culture. The examined additives were carbon sources (glucose, maltose, rice starch, sucrose, and corn starch; at $1 \% \mathrm{w} / \mathrm{w})$; nitrogen sources (urea, yeast extract, tryptone, tryptic soy, peptone, nutrient broth, skim milk, casamino acids, soytone, malt extract, $\mathrm{NH}_{4} \mathrm{Cl}$, and $\mathrm{NH}_{4} \mathrm{NO}_{3}$ at $1 \%$ w/w), metal salts $\left(\mathrm{CaCl}_{2}, \mathrm{MgCl}_{2}, \mathrm{MnCl}_{2}, \mathrm{CuSO}_{4}, \mathrm{CoSO}_{4}, \mathrm{FeSO}_{4}, \mathrm{ZnSO}_{4}\right.$, and $\mathrm{KCl}$ at $2.5 \mathrm{mM}$ ), surfactant (Tween 20, Tween 80, Triton $\mathrm{X}-100$ at $0.05 \% \mathrm{v} / \mathrm{w}$, and sodium dodecyl sulfate [SDS] and ethylenediaminetetraacetic acid [EDTA] at $0.4 \mathrm{mM}$ ). SSF process was carried out at $30^{\circ} \mathrm{C}$ for 3 days with wheat bran containing 33\% initial moisture, which was inoculated with $10 \%(\mathrm{v} / \mathrm{w})$ of a 1-day-old seeding culture. Each experiment was replicated three times.

\section{Results and Discussion}

Improvement of fungal strain producing cellulase. Cellulase-hyper producing Aspergillus sp. SU14 was selected by $\gamma$ - and UV-irradiation, and NTG treatment described earlier. The cellulose activity of the selected SU-M15 mutant was $19.2 \mathrm{U} / \mathrm{g}$, which represented a 2-fold improved activity than that of wild type $(9.65 \mathrm{U} / \mathrm{g})$ (Fig. 1). The success of the presently-employed mutant selection conditions has been amply demonstrated. For example, Thermomyces lanuginosus treated with three cycles of UV and NTG elevated $\alpha$-amylase and glucosamylase by 7 -fold and 3-fold, respectively, as compared to the wild type, in one study [17]. In another study, F. oxysporum successively treated with UV and NTG displayed 3-fold elevated cellulose activity than wild type [11].

\section{Optimal conditions for SSF.}

Substrate: Selection of a suitable substrate and physical, chemical and biochemical process parameters are cru- 


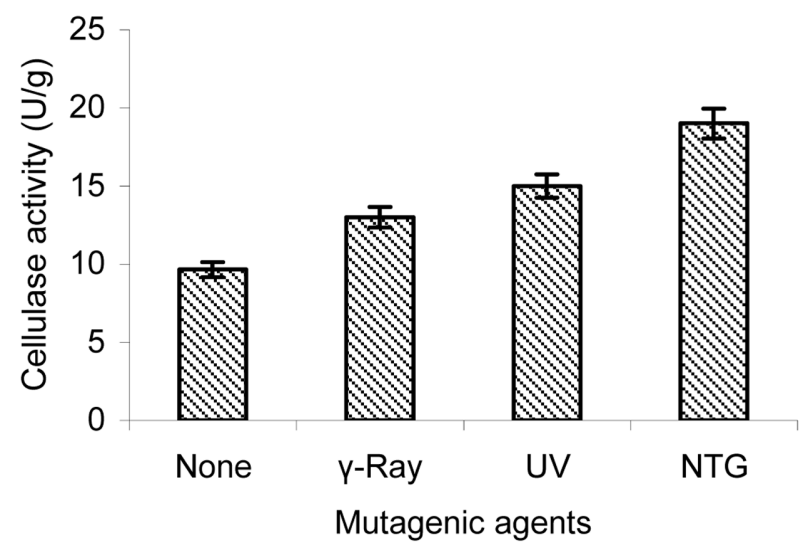

Fig. 1. Cellulase production on several solid media by Aspergillus sp. SU14 wild type and mutant strain after treatment with $\gamma$-ray, ultraviolet (UV), and N-methyl$\mathrm{N}$ '-nitro-N-nitrosoguanidine (NTG). None, wild type; $\gamma$-ray, selected strain after treatment with $\gamma$-ray; UV, selected strain after treatment with $\gamma$-ray followed by UV radiation; NTG, selected strain (Aspergillus sp. SU-M15) after treatment with $\gamma$-ray and UV radiation, followed by NTG.

cial in optimizing SSF. Presently, wheat ban resulted in a favorably high production of cellulase $(19.2 \mathrm{U} / \mathrm{g})$, while other solid media such as husk, sawdust did not (Fig. 2A). Wheat bran also was the best of the various solid sub- strates used for the colonization of Aspergillus sp. SUM15, as indicated by the maximum visible growth and the highest enzyme yield (Fig. 2A). Therefore, solid wheat bran was chosen for further experiments.

Initial moisture content in the solid substrate: Cellulase production depends on the moisture level in the solid substrate. A 50\% moisture content resulted in cellulose production $(76.6 \mathrm{U} / \mathrm{g})$ that was much higher than obtained at other moisture levels (Fig. 2B). Moisture contents $<40 \%$ or $>55 \%$ were not suitable for high cellulase production. In SSF, moisture level plays an important role in biosynthesis and secretion of many kinds of enzymes, especially cellulases. Very high moisture content in solid medium results in decreased substrate porosity as well as reducing oxygen penetration among the substrate particles [18], but excessively low moisture levels in solid medium leads to poor microbial growth, poor development and poor accessibility to nutrients [18].

Incubation temperature: Temperature strongly affects the SSF process, which varies according to the organism involved. Even slight changes in temperature can affect cellulase production. Presently, the optimal temperature for the highest production of cellulase was $35^{\circ} \mathrm{C}$, with cellulase production decreasing at higher temperatures (Fig. 2C).

Medium pH: The optimal $\mathrm{pH}$ varies with different microorganisms and enzymes. Presently, the highest production
A

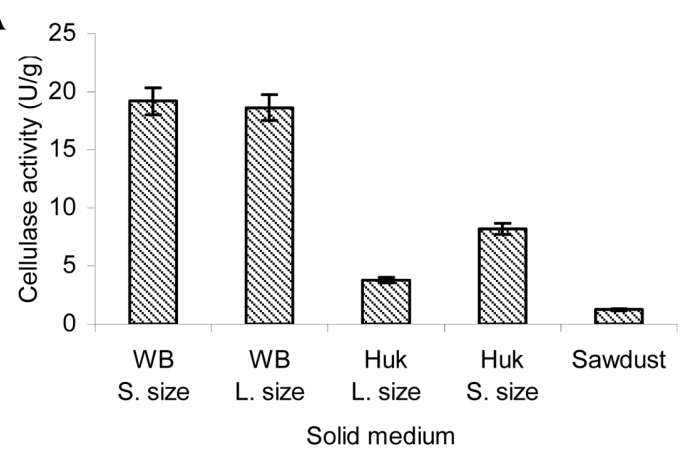

C

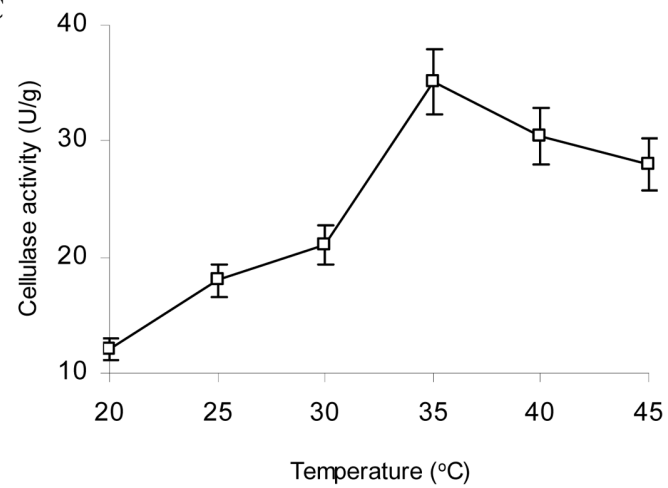

B

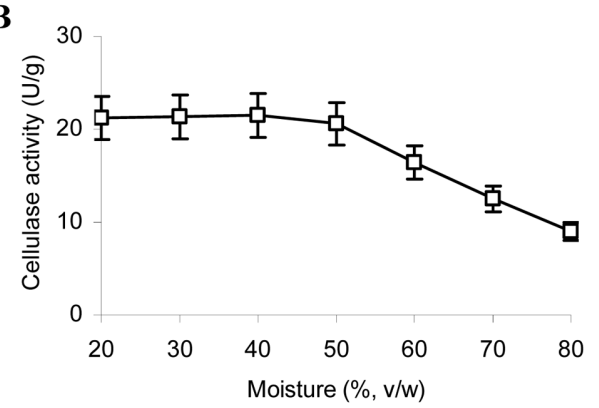

D

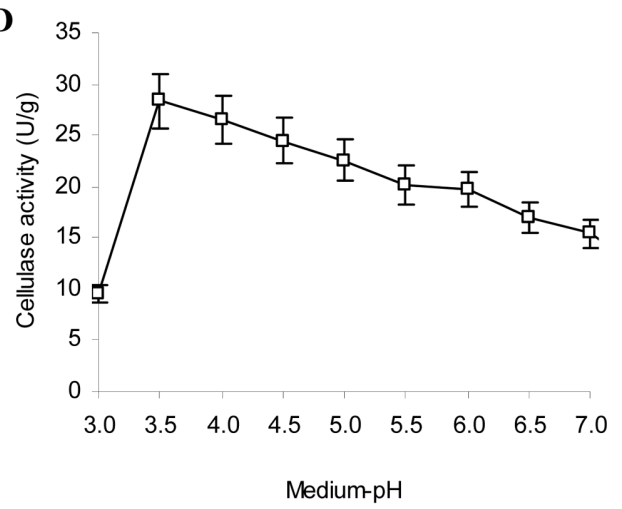

Fig. 2. Effect of various culture conditions on cellulase production. A, Enzyme production in different solid substrates; B, Effect of initial moisture levels on cellulase production; $\mathrm{C}$, Effect of incubation temperature on cellulase production; D, Effect of pH of solid medium on cellulase production. WB, wheat bran; L. size, large size; S. size, small size; Huk, husk. 
of cellulase was observed at a $\mathrm{pH}$ of 3.5 (Fig. 2D). The influence of $\mathrm{pH}$ on cellulase production highlighted the widely-known importance of $\mathrm{pH}$ for microbial growth and metabolic activities, and the sensitivity of the latter to $\mathrm{pH}$ change.

Fermentation time: The highest production of enzyme $(28.31 \mathrm{U} / \mathrm{g})$ was observed after 3 days of fermentation (Fig. 3A).

Aeration area: The quantity of moistened wheat bran per flask volume of culture markedly affected the porosity and aeration of the medium. Production of cellulase was greatest $(29.6 \mathrm{U} / \mathrm{g})$ at a substrate mass : flask volume ratio of $3: 100$ (Fig. 3B). This ratio was used for further experiments.

Age of seeding culture: A seeding culture age of 2 days was suitable for inoculation and resulted in the high production of cellulase $(30.5 \mathrm{U} / \mathrm{g})$. Use of seeding cultures more than 2-day-old produced a rapid decrease of cellulase production (Fig. 3C).

Inoculum size: Cellulase production increased together with an increasing of initial inoculum size ranging from $10 \sim 50 \%(\mathrm{v} / \mathrm{w})$ of total inoculated solid medium. Maximum cellulase production $(32.34 \mathrm{U} / \mathrm{g})$ was observed at an inoculum size of $26 \%(\mathrm{v} / \mathrm{w})$ (Fig. 3D). This inoculum size was employed for further experiments. The results highlighted the importance of inoculum density in SSF. Higher inoculum levels increased the spore number per gram of

A

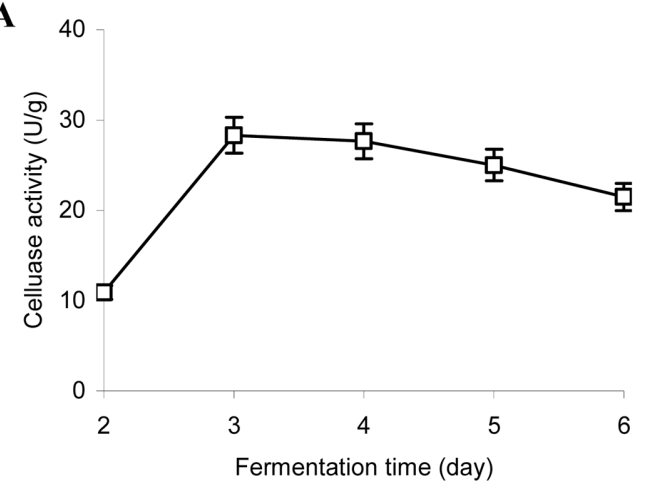

C

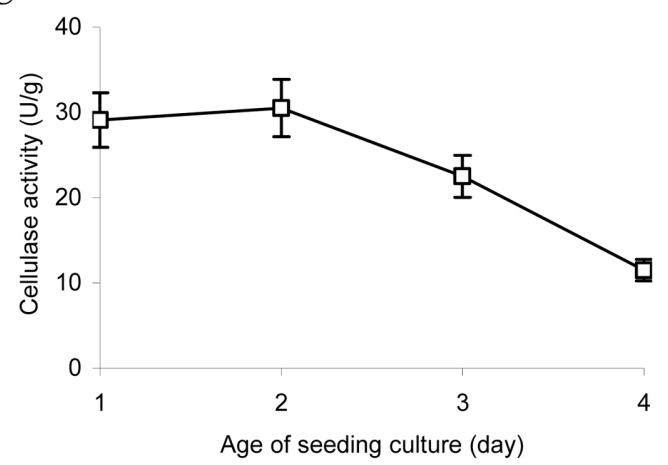

solid substrate, as well as the water content in the medium; both factors acted to hinder the penetration of oxygen into the solid medium, inhibiting fungal growth and cellulase production. However, a lower inoculum size of seeding culture extended the time required for the fermentation process to complete a batch culture.

Effect of medium additives on the enzyme production.

Carbon: Glucose, corn starch, sucrose, maltose or rice starch was supplemented to the basal medium, each at $1 \%$ (v/w). Each additive differentially affected cellulase production. Rice starch and corn starch slightly enhanced cellulase production, while the other carbon sources did not enhance cellulase production (Fig. 4A).

Nitrogen: $\mathrm{NH}_{4} \mathrm{Cl}$, urea, and malt extract significantly enhanced the production of cellulase, while skim milk markedly decreased cellulase production (Fig. 4B).

Surfactants: Surfactants and fatty acids can enhance the production of hydrolytic enzymes [19]. Surfactants are crucial in enhancing microbial growth in SSF by promoting the penetration of water into the solid substrate matrix, leading in an increase of surface area [20]. Presently, amendment of wheat bran with any of the tested surfactants (Tween 20, Tween 80, Triton X100, EDTA and SDS) enhanced cellulase production. Tween 80 and EDTA were most effective, and resulted in cellulase production of 38 40 U/g (Fig. 4C).
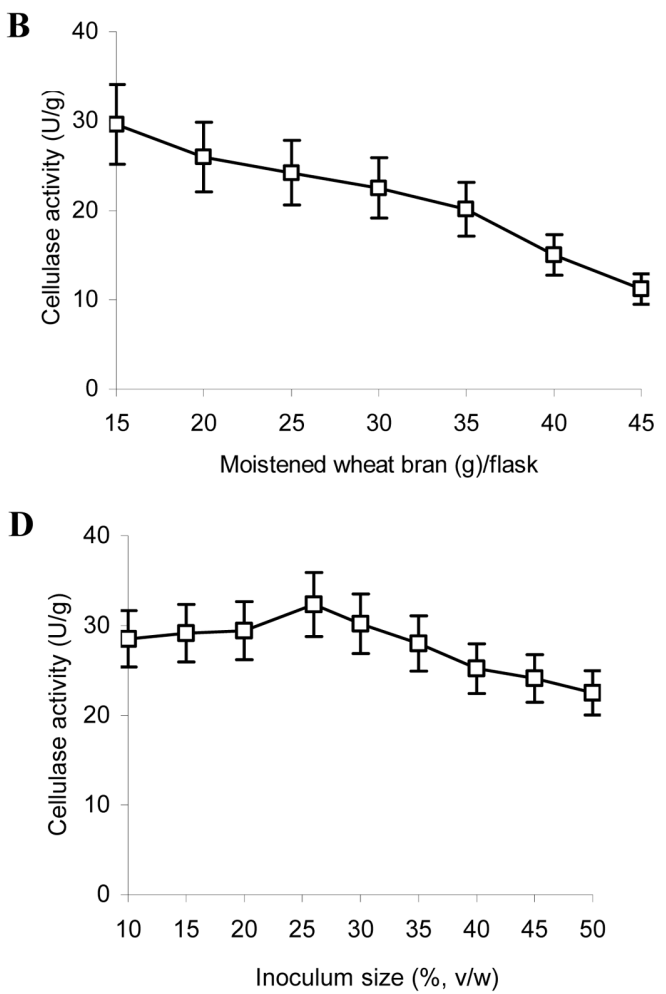

Fig. 3. Effect of various culture conditions on cellulase production. A, Fermentation time; B, Aeration area (moistened wheat bran/ flask); C, Age of liquid-seeding culture; D, Inoculum size. 
A

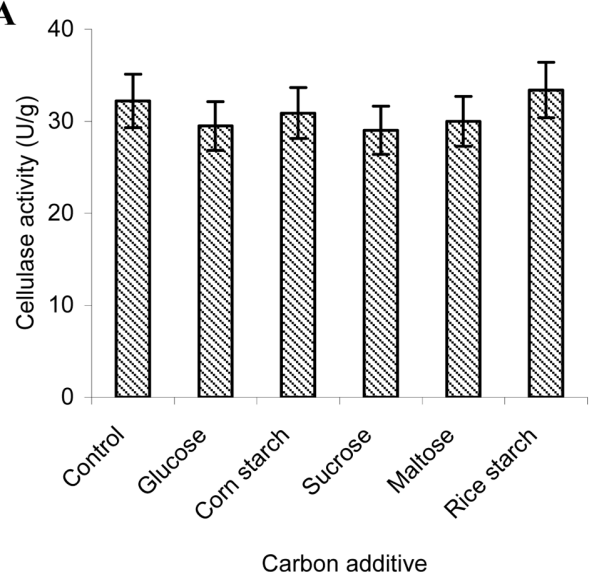

C

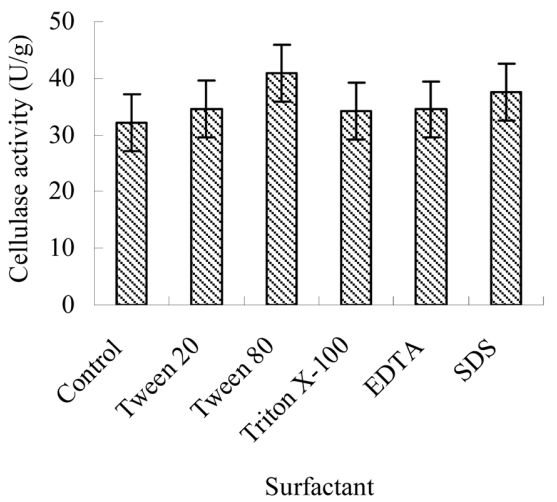

B

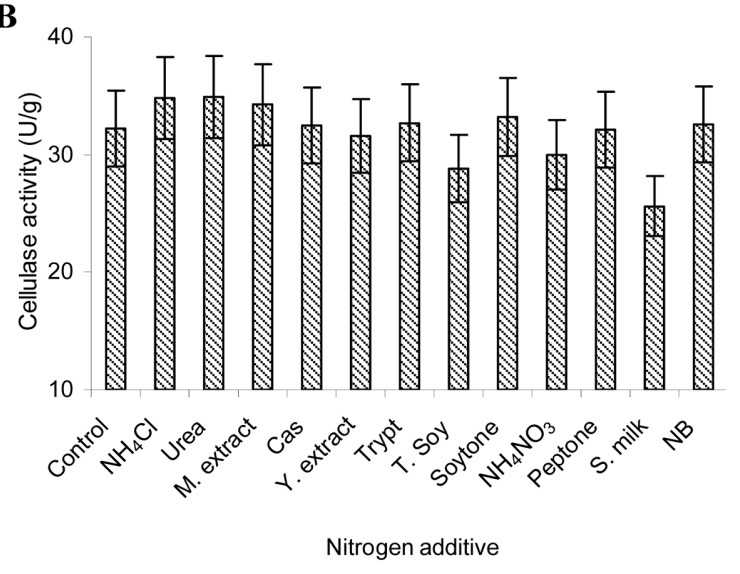

D

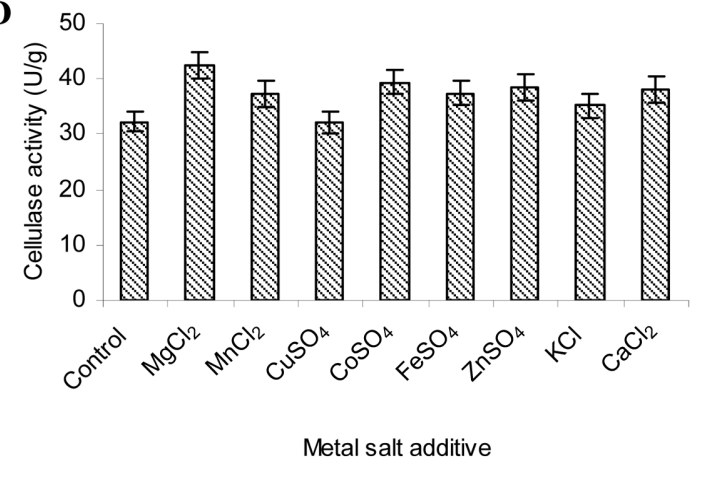

Fig. 4. Effect of medium additives on cellulase production. A, Carbon; B, Nitrogen; C, Surfactant; D, Metal salt. M. extract, malt extract; Cas, casamino acids; Y. extract, yeast extract; Trypt, tryptone; T. Soy, Tryptic soy; S. milk, skim milk; NB, nutrient broth; EDTA, ethylene diamine tetraacetic acid; SDS, sodium dodecyl sulfate.

Metal salts: The influence of metal salts on fermentative metabolism is not well-documented. $\mathrm{CuSO}_{4}$ is an essential salt for some organisms [21]. However, presently all tested metal salts enhanced cellulase production, except $\mathrm{CuSO}_{4}$ (Fig. 4D). Solid medium supplemented with $\mathrm{MgCl}_{2}$ produced the highest yield of cellulase $(43 \mathrm{U} /$ g) (Fig. 4D).

Production of cellulase in supplemented medium at optimal conditions. Cellulase was produced by Aspegillus sp. SU-M15 using SSF from wheat-bran using the aforementioned optimized parameters. Cellulase production was $82.50 \mathrm{U} / \mathrm{g}$ after $72 \mathrm{hr}$, which was an 8.5 -fold increase over the wild-type (Fig. 5).

In conclusion, a mutant fungal strain was created by repeated and sequential mutagenesis with $\mathrm{Co}^{60} \gamma$-rays, UV radiation and NTG. From the resulting 500 mutants, Aspergillus sp. SU-M15 was selected. SU-M15 was cultivated in SSF for the production of cellulase using wheat bran as the substrate. Under the deduced optimized medium and SSF conditions, the cellulase production of SU-M15 was $82.5 \mathrm{U} / \mathrm{g}$, representing an 8.5 -fold increase in cellulase production than that produced in wheat bran basal medium by the wild type strain.

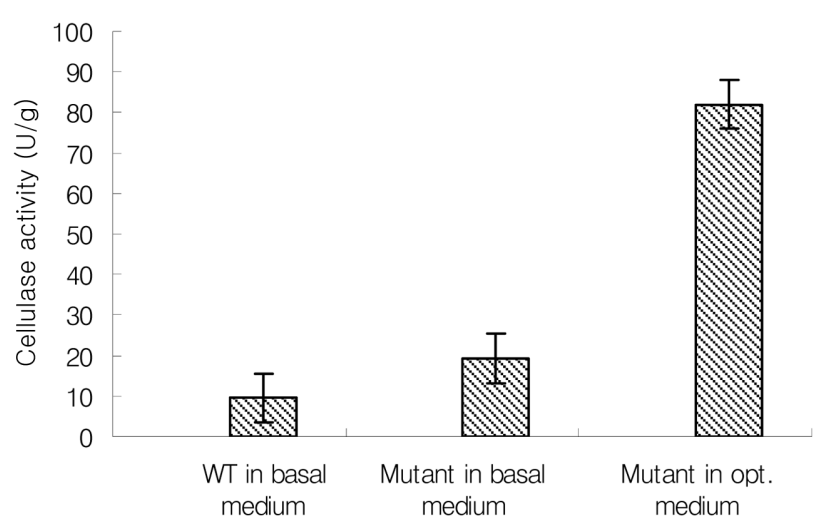

Fig. 5. Production of cellulase in basal solid medium and supplemented solid medium. WT in basal medium, cellulase production by wild type strain on basal medium; Mutant in basal medium, cellulase production by mutant Aspergillus sp. SU-M15 in basal medium; Mutant in opt. medium, cellulase production by mutant Aspergillus sp. SU-M15 in optimal mediumcondition.

\section{Acknowledgements}

This study was supported by the Technology Develop- 
ment Program (309016-5) of Ministry for Food, Agriculture, Forestry and Fisheries, Republic of Korea.

\section{References}

1. Duff SJ, Murray WD. Bioconversion of forest products industry waste cellulosics to fuel ethanol: a review. Bioresour Technol 1996;55:1-33.

2. Jones RP, Pamment N, Greenfield PF. Alcohol fermentation by yeasts: the effect of environmental and other variables. Process Biochem 1981;16:42-9.

3. Rogers PL, Lee KJ, Tribe DE. High productivity ethanol fermentation with Zymomonas mobilis. Process Biochem 1980;15:7-11.

4. Shin D, Yoo A, Kim SW, Yang DR. Cybernetic modeling of simultaneous saccharification and fermentation for ethanol production from steam-exploded wood with Brettanomyces custersii. J Microbiol Biotechnol 2006;16:1355-61.

5. Durand H, Clanet M, Tiraby G. Genetic improvement of Trichoderma reesei for large scale cellulase production. Enzyme Microb Technol 1988;10:341-6.

6. Kang SW, Park YS, Lee JS, Hong SI, Kim SW. Production of cellulases and hemicellulases by Aspergillus niger KK2 from lignocellulosic biomass. Bioresour Technol 2004;91: 153-6.

7. Sohail M, Siddiqi R, Ahmad A, Khan SA. Cellulase production from Aspergillus niger MS82: effect of temperature and pH. New Biotechnol 2009;25:437-41.

8. Sun Y, Cheng JY. Hydrolysis of lignocellulosic materials for ethanol production: a review. Bioresour Technol 2002;83:111.

9. Zhang YH, Himmel ME, Mielenz JR. Outlook for cellulase improvement: screening and selection strategies. Biotechnol Adv 2006;24:452-81.

10. Parekh S, Vinci VA, Strobel RJ. Improvement of microbial strains and fermentation processes. Appl Microbiol Biotechnol 2000;54:287-301.
11. Kuhad RC, Kumar M, Singh A. A hypercellulolytic mutant of Fusarium oxysporum. Lett Appl Microbiol 1994;19:397400.

12. $\mathrm{Vu} \mathrm{VH,} \mathrm{Pham} \mathrm{TA,} \mathrm{Kim} \mathrm{K.} \mathrm{Fungal} \mathrm{strain} \mathrm{improvement} \mathrm{for}$ cellulase production using repeated and sequential mutagenesis. Mycobiology 2009;37:267-71.

13. Pandey A. Solid-state fermentation. New Delhi: Wiley Eastern Limited; 1994. p. 12-7.

14. Babu KR, Satyanarayana T. $\alpha$-Amylase production by thermophilic Bacilluscoagulans in solid state fermentation. Process Biochem 1995;30:305-9.

15. Grajek W. Comparative studies on the production of cellulases by thermophilic fungi in submerged and solid-state fermentation. Appl Microbiol Biotechnol 1987;26:126-9.

16. Miller GL. Use of dinitrosalicylic acid reagent for determination of reducing sugar. Anal Chem 1959;31:426-8.

17. Rubinder K, Chadha BS, Singh N, Saini HS, Singh S. Amylase hyperproduction by deregulated mutants of the thermophilic fungus Thermomyces lanuginosus. J Ind Microbiol Biotechnol 2002;29:70-4.

18. $\mathrm{Vu} \mathrm{VH}$, Pham TA, Kim K. Improvement of a fungal strain by repeated and sequential mutagenesis and optimization of solid-state fermentation for the hyper-production of rawstarch-digesting enzyme. J Microbiol Biotechnol 2010;20: 718-26.

19. Singh A, Abidi AB, Darmwal NS, Agrawal AK. Influence of nutritional factors of cellulase production from natural lignocellulosic residues by Aspergillus niger. Agric Biol Res 1991; 7:19-27.

20. Asgher M, Asad MJ, Legge RL. Enhanced lignin peroxidase synthesis by Phanerochaete chrysosporium in solid state bioprocessing of a lignocellulosic substrate. World J Microbiol Biotechnol 2006;22:449-53.

21. Fraústo da Silva JJ, Williams RJ. The biological chemistry of the elements: the inorganic chemistry of life. New York: Clarendon Press; 1993. 